\title{
Evidências da relação entre a frequência no ensino infantil e o desempenho dos alunos do ensino fundamental público no Brasil
}

\author{
Walcir Soares da Silva Junior \\ Flávio de Oliveira Gonçalves ${ }^{\star \star}$
}

\begin{abstract}
Este artigo tem por objetivo estimar o impacto da frequência ao ensino infantil na proficiência dos alunos do ensino fundamental público no Brasil, utilizando como proxy as notas dos alunos em português e matemática disponíveis na Prova Brasil 2011. Foi empregada a metodologia de pareamento nos escores de propensão sob a hipótese de seleção por variáveis observáveis, que tem por finalidade encontrar um contrafactual. Para controlar as características relacionadas ao desempenho, foram estimadas regressões lineares pelo método duplamente robusto dos mínimos quadrados ponderados pelos escores de propensão. Encontraram-se efeitos positivos e significantes estatisticamente sobre as notas de português e matemática do $5^{\circ}$ e e $9^{\circ}$ anos para alunos que frequentaram a pré-escola e a creche em comparação com aqueles que iniciaram os estudos diretamente no ensino fundamental.
\end{abstract}

Palavras-chave: Ensino infantil. Propensity score matching. Mínimos quadrados ponderados pelo propensity score.

\footnotetext{
*Universidade Federal do Paraná (UFPR), Curitiba-PR, Brasil (contatodabliu@gmail.com).

** Universidade Federal do Paraná (UFPR), Curitiba-PR, Brasil (f.goncalves@ufpr.br).
} 


\section{Introdução}

A primeira infância tem tido grande destaque nos estudos sobre educação e desenvolvimento de habilidades tanto cognitivas quanto não cognitivas (CURI; MENEZES-FILHO, 2006). O foco nos programas educacionais da primeira infância enquanto políticas públicas mais eficientes vem da evidência de que esta fase parece ser um período sensível e crítico ${ }^{1}$ na produção de algumas habilidades importantes, como por exemplo o QI, que se estabiliza aos dez anos de idade (CUNHA et al., 2006).

Apesar das diversas evidências na literatura a favor da primeira infância, existem poucos estudos experimentais ou quase experimentais avaliando programas de larga escala, principalmente para dados brasileiros. Desse modo, justifica-se a estimação de evidências que sirvam de suporte para estratégias de política pública que visem o aumento e melhoria do ensino infantil. Tendo em vista que o sistema educacional brasileiro possui particularidades, a simples generalização de estudos internacionais pode não ser adequada.

Determinar causalidade é hoje um dos principais desafios na literatura de economia da educação, que vai além de encontrar simples correlação entre variáveis educacionais e quaisquer resultados importantes tanto no âmbito acadêmico quanto no mercado de trabalho. Existem duas principais características desejáveis nas pesquisas de avaliação de impacto. A primeira é a realização de estudos experimentais, em que são selecionados grupos de controle e tratamento aleatorizados, possibilitando medir o efeito causal de uma política ou programa em determinado grupo isoladamente. A segunda é a disponibilidade de dados longitudinais que contemplem informações em nível micro para mais de um período.

Para dados educacionais, entretanto, isso pode ser bastante complicado, seja pelas implicações éticas de se selecionar um grupo para determinado programa em detrimento de outro, seja pelos altos custos desse tipo de experimento, bem como pela confidencialidade dos dados que não permitem seguir um mesmo aluno por mais de um período. Entretanto, algumas metodologias preocupadas em criar artificialmente esse ambiente experimental podem ser usadas para se tentar, na medida da possibilidade dos dados, inferir causalidade, como é o caso deste trabalho.

O objetivo deste artigo é medir o impacto do ensino infantil sobre as habilidades cognitivas dos alunos no ensino fundamental público no Brasil. Como proxy para habilidades cognitivas foram utilizados os resultados dos testes padronizados de português e matemática, disponíveis na Prova Brasil 2011, para o 5ํㅜ e 9ํanos do ensino fundamental. A hipótese é de que o ensino infantil tenha importante contribuição no aumento das habilidades cognitivas dos alunos no ensino fundamental.

Sob a suposição de existência de viés de seleção por algumas variáveis observáveis, utilizou-se um estimador de propensity score matching (PSM), que tem por objetivo criar um contrafactual, ou seja, um grupo de alunos comparáveis com relação a três variáveis

\footnotetext{
$\overline{1}$ Esses conceitos são detalhados na seção 2.
} 
teóricas que parecem afetar essa escolha: renda dos pais; escolaridade dos pais; e disponibilidade de alguém em casa para cuidar da criança. ${ }^{2}$

Existem ainda outras características que parecem importantes nesta seleção, como a disponibilidade de creche ou escolas nas proximidades da residência da criança, o interesse ou valorização dos pais pela educação de seus filhos e a qualidade da educação. No entanto, essas variáveis ou não são diretamente observáveis, ou não estão disponíveis no nível do aluno, representando um limite deste trabalho. Outro limite seria a necessidade de utilizar dados longitudinais que acompanhassem os mesmos alunos em anos diferentes. No entanto, os dados educacionais do Instituto Nacional de Estudos e Pesquisas Educacionais Anísio Teixeira (Inep), por motivos de confidencialidade, não disponibilizam a junção dessas informações em nível do aluno.

Além do método de pareamento para se tentar controlar a seleção dos alunos, uma das principais contribuições deste trabalho é aliar um método quase experimental com outro duplamente robusto: a regressão linear por mínimos quadrados ponderados pelo propensity score, que controla, além das variáveis do processo de seleção (contidas no escore de propensão), variáveis responsáveis pelo desempenho, tais como raça, se o aluno reprovou ou não, se sempre estudou em escola pública, etc. ${ }^{3}$ Ambas as metodologias - propensity score matching e mínimos quadrados ponderados pelo propensity score - encontraram efeitos positivos e estatisticamente significativos da pré-escola e da creche nas notas dos alunos de $5^{\circ}$ e 90 anos do ensino fundamental.

É importante mencionar que, no Brasil, entre 2000 e 2010, segundo dados do Inep, os investimentos em educação infantil ficaram estagnados em $0,4 \%$ ao ano do Produto Interno Bruto, enquanto os investimentos totais em educação aumentaram $23 \%$ durante estes dez anos, passando de 4,7\% para 5,8\% do PIB. Dados do IBGE (2012) mostram que, em 2011, apenas 55,2\% das crianças de quatro anos estavam frequentando a creche ou a pré-escola, proporção que diminui para $20,8 \%$ entre aquelas de até três anos.

Ainda segundo dados do Inep, a educação infantil é o nível educacional com menor percentual do investimento público direto, registrando inclusive uma redução de $10 \%$ na última década, além de continuar representando o menor nível no investimento direto em educação por estudante, ${ }^{4}$ com um aumento de aproximadamente $70 \%$, entre 2000 e 2010 , enquanto os investimentos agregados em educação cresceram cerca de $120 \%$, nesse período. Assim, a etapa da educação que autores como Curi e Menezes-Filho (2006), Heckman (2000), Cunha et al. (2006), Carneiro e Heckman (2003) e outros evidenciam como uma das mais importantes no desenvolvimento de habilidades do ciclo de ensino tem tido pouco investimento em relação aos ensinos fundamental, médio e superior no Brasil.

\footnotetext{
${ }^{2}$ A terceira seção apresenta um breve modelo teórico que detalha melhor a relação destas variáveis com a seleção.

${ }^{3}$ Essas variáveis serão mais bem detalhadas na seção da estratégia empírica.

${ }^{4}$ É preciso salientar que aqui o investimento por estudante utilizado é a média do país. Existe um problema muito mais complexo quanto à desigualdade na distribuição dos recursos, o que faz com que, em municípios mais ricos, o investimento seja maior.
} 


\section{A relevância do ensino público infantil no Brasil}

A educação infantil no Brasil é dividida em creche, dos 0 aos 3 anos, e pré-escola, de 4 a 5 anos. ${ }^{5}$ A partir da década de 1990 (Constituição de 1988), o acesso a esse nível de ensino teve sua importância ampliada, pois, além de se prever um aumento da quantidade educacional às crianças de 0 a 6 anos, a educação infantil passou a ser tratada como um direito da criança.

Em meados de 1990, aliada à preocupação com a qualidade educacional como um todo, a qualidade da educação infantil também passou a ganhar relevância, principalmente após a promulgação da Lei de Diretrizes e Bases da Educação Nacional (LDB), em 1996, que incluiu o atendimento de crianças de 0 a 3 anos nas creches (GUIMARÃES; PINTO, 2001). Campos (1997) explica que a importância da educação infantil mudou ao longo do tempo para diversos níveis. Desse modo, o aumento da literatura sobre educação infantil nos últimos anos e, por conseguinte, a ampliação das evidências de que os retornos nessa fase da infância são muito altos fazem com que a importância do ensino infantil seja crescente.

Quando se trata de habilidades, a literatura comumente refere-se às habilidades cognitivas e não cognitivas, ${ }^{6}$ responsáveis pelo sucesso futuro da criança, que podem ser produzidas tanto pela família - importância especial para o papel da mãe - como pelas ações pessoais do indivíduo e sua interação com o ambiente. ${ }^{7}$ Assim, políticas públicas podem agir sobre o componente advindo do ambiente, já na fase da primeira infância, aumentando a produtividade dos investimentos educacionais nos períodos posteriores (HECKMAN, 2000; CARNEIRO; HECKMAN, 2003).

O processo educacional, portanto, já não é mais visto como composto por uma só fase, mas sim por diversas fases em que o ser humano forma suas habilidades. A primeira infância possui um papel fundamental, pois, nesta fase, se algumas habilidades não forem desenvolvidas, tentar desenvolvê-las por meio de investimentos tardios pode ser muito mais custoso e pouco eficiente (HECKMAN, 2000; CARNEIRO; HECKMAN, 2003; CUNHA et al., 2006).

Knudsen (2004) separa o processo de aprendizado em dois períodos: o crítico e o sensível. Segundo o autor, o cérebro é amplamente influenciado pelas experiências do indivíduo. Quando o efeito de determinada experiência é muito forte em um período limitado, esse é chamado de período sensível. Quando certa experiência é crucial nesse período para o desenvolvimento normal do indivíduo, e altera o seu desempenho permanentemente, esse período é chamado de crítico.

\footnotetext{
${ }^{5}$ Recentemente, o ensino fundamental foi aumentado em um ano, considerando-se então o que era o ensino pré-escolar como primeiro ano. Assim, a pré-escola passou de 4 a 6 anos para 4 a 5 anos de idade.

${ }^{6}$ Habilidades cognitivas, basicamente, são aquelas ligadas à inteligência e à organização, como, por exemplo, memória, capacidade de abstração, etc. Já as não cognitivas são aquelas ligadas às competências socioemocionais, como motivação, autonomia, equilíbrio emocional, etc.

${ }^{7}$ Este trabalho foca no impacto sobre as habilidades cognitivas, que têm como proxy as notas dos alunos na Prova Brasil 2011. No entanto, evidências mostram que as habilidades não cognitivas, mais difíceis de mensurar, contribuem tanto quanto as cognitivas nos resultados educacionais (CUNHA et al., 2006).
} 
Um exemplo disso é o desenvolvimento do QI, principal medida de habilidade cognitiva, que estabiliza em torno dos dez anos de idade. Desse modo, a primeira infância é um período sensível e crítico no desenvolvimento desta habilidade (KNUDSEN, 2004).

Heckman (2000) e Carneiro e Heckman (2003) reconhecem que as habilidades, tanto cognitivas quanto não cognitivas, possuem um componente genético e outro influenciado pelo ambiente. Para Heckman (2008), evidências da epigenética sugerem que a distinção entre genética e ambiente é obsoleta, assim como a prática de discernir entre resultados "nature" e "nurture", ou seja, o que é da natureza e o que é estimulado pelo ambiente externo.

Segundo Rutter $^{8}$ (2006 apud HECKMAN, 2008), existe uma extensa literatura mostrando que a interação entre os dois componentes é central para explicar o desenvolvimento humano e animal. Autores como Caspi, Williams, Kim-Cohen et al. (2007) mostraram que o desenvolvimento intelectual das crianças é influenciado tanto por fatores genéticos quanto ambientais. Turkheimer, Haley, Waldron et al. (2003) encontraram um importante papel para o ambiente na determinação do QI.

Outra característica importante do capital humano é exibir autoprodutividade e complementaridade. Autoprodutividade porque a realização de habilidades num estágio inicial aumenta a realização de outras habilidades no futuro. Complementaridade porque investimentos mais tarde não são produtivos e eficientes se o indivíduo não possui uma base de habilidades fundamentais produzida na primeira infância. Em resumo, se a base é fraca, o retorno para investimentos tardios é baixo (CUNHA et al., 2006).

As evidências empíricas corroboram esses conceitos. Mais do que a falta de recursos, que é um dos maiores problemas em educação no mundo todo, não ter feito o ensino infantil, para um estudo em alunos norte-americanos, é o que mais explica a conclusão do ensino médio e ingresso no ensino superior. Aqueles que não frequentaram o ensino infantil possuem um déficit de habilidades que explica sua baixa escolaridade (CARNEIRO; HECKMAN, 2002, 2003).

Em outro estudo, Cunha et al. (2006) apresentam um exercício em que mostram que a diferença racial e de renda existente em testes de desempenho é significantemente reduzida quando se controlam algumas características como educação materna, habilidades cognitivas e estrutura familiar. Assim, os autores concluem que programas de enriquecimento de ambiente inicial para crianças em desvantagem podem parcialmente remediar a diferença entre estas e as crianças mais favorecidas.

Um estudo de experimento natural sobre um programa de ensino infantil de construções de escolas na Argentina concluiu que os testes de aptidão (habilidades cognitivas) apresentam uma melhora de $8 \%$ para cada ano de pré-escola, além de melhoras nas variáveis atenção, esforço, participação e disciplina (habilidades não cognitivas) (BERLINSKI et al., 2006). Feinstein et al. (1998) apresentam resultados positivos, válidos também

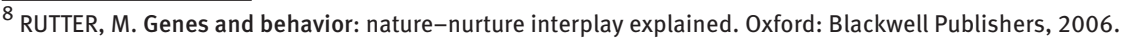


após controlar educação dos pais, classe social e interesse dos pais e vizinhança, de que crianças norte-americanas dos 7 aos 11 anos demonstram maior desempenho na escola se frequentaram uma pré-escola ou ficaram sob os cuidados da mãe.

Existem alguns estudos para o Brasil, no entanto, a literatura de avaliação de impacto com métodos experimentais ou quase experimentais ainda é bastante limitada. Curi e Menezes Filho (2006) encontraram efeitos positivos e significativos de ter frequentado a creche sobre a probabilidade de conclusão das etapas de ensino e salários futuros. Britto (2012), utilizando um painel de escolas extraído dos microdados da Prova Brasil 2009 e controlando para características específicas dos indivíduos e do background familiar, verificou que as escolas possuem um desempenho melhor se seus alunos ingressaram no ensino infantil, em comparação com escolas em que os alunos entraram diretamente no ensino fundamental.

Campos et al. (2011), utilizando análise hierárquica, observaram que alunos que frequentaram a pré-escola de boa qualidade, quando comparados a seus colegas que não frequentaram uma pré-escola, obtiveram notas 2,9 pontos mais altas, correspondendo a um acréscimo de $12 \%$ na escala de notas da Prova Brasil. Os autores verificaram também que escolaridade da mãe, renda familiar e o Índice de Desenvolvimento da Educação Básica (Ideb) da escola são fatores importantes nos resultados apresentados nessa avaliação.

Felício e Vasconcellos (2007), empregando dados do Saeb 2003, estimaram com base nas informações individuais dos alunos, aplicando o método de propensity score matching (PSM), que frequentar a escola antes da primeira série tem um impacto positivo e significativo sobre a proficiência das crianças em testes padronizados de matemática, no $5^{0}$ ano. A diferença deste trabalho para o exercício de Felício e Vasconcellos (2007), além da atualização da evidência, é utilizar juntamente com o pareamento um método duplamente robusto para controlar as características relacionadas ao desempenho, bem como um modo de seleção com melhor ajuste.

Existem ainda alguns importantes projetos de intervenção na primeira infância com estudos experimentais e longitudinais devidamente aleatorizados e com grupos de controle e tratamento, executados em escolas dos Estados Unidos, o Perry Preschool, o Abecedarian Program e o Star Project. Todos eles tinham como pressuposto um programa de ensino infantil em tempo integral de alta qualidade e acompanhamento especial, além do objetivo de seguir os alunos até a idade adulta para avaliar os resultados nos grupos de controle e tratamento.

Para o Perry Preschool, segundo Heckman (2008), foram identificados padrões consistentes de altos resultados para o grupo de tratamento em comparação com o grupo de controle, como maiores pontuações nos testes, maior nível de escolaridade e menor necessidade de educação especial. De acordo com Barnett (2004), quando atingiram a idade dos 40 anos, os indivíduos do grupo de tratamento tinham os maiores níveis salariais e de casa própria, além de não precisarem de auxílios governamentais. 0 Abecedarian Program resultou em um aumento permanente no QI dos participantes e o Projeto Star mostrou uma 
diferença positiva na cognição para os grupos de classes de tamanhos menores e maiores ganhos salariais no futuro, além de melhor comportamento em sala de aula em comparação com o grupo de controle.

Mas, apesar dessas evidências empíricas, a evidência causal de impacto de programas aplicados na pré-escola para crianças em desvantagens nos resultados de longo prazo ainda é bastante limitada. É difícil afirmar que, se aplicados os mesmos programas em outro país ou contexto em que o sistema educacional esteja em outro estágio, como no caso do Brasil, os resultados serão os mesmos. 0 uso de dados longitudinais, no caso destes estudos norte-americanos, também foi muito importante na avaliação dos mesmos, mas esses foram programas pontuais de escala limitada em escolas que diferem em diversos aspectos das escolas brasileiras. Ou seja, o grande problema é a replicabilidade destes resultados em programas de larga escala, que é, em última instância, o objetivo de interesse efetivo da política pública.

Desse modo, justifica-se a contribuição de trabalhos como esse, apresentando evidências de políticas de larga escala como é o caso do ensino infantil no Brasil, utilizando métodos quase experimentais que se aproximam de experimentos aleatórios metodologicamente e dão alguma evidência causal dos efeitos destas políticas.

Seguindo o argumento de Heckman e Masterov (2007), é preciso atentar para o fato de que essa discussão em favor das políticas de melhoria nos ambientes iniciais das crianças em desvantagem não quer dizer que todas as habilidades e motivações são formadas nos primeiros anos. Muito menos quer dizer que as escolas e empresas não importam na formação das pessoas, ou que os anos iniciais são os únicos determinantes do sucesso.

Essas evidências defendem o papel que o ambiente inicial tem nos resultados posteriores e que sua importância é negligenciada nas políticas atuais. Inclusive, as evidências da tecnologia de formação das habilidades humanas dizem que ambientes inicialmente enriquecidos precisam ser seguidos por boa escolaridade e aprendizagem no local de trabalho, ou seja, a complementaridade está intrínseca no processo de formação das habilidades.

Desse modo, intervenções iniciais promovem eficiência econômica e reduzem as desigualdades, ou seja, não há trade-off para investimentos na primeira infância. Já para intervenções de remediação para adolescentes em desvantagem, aqueles que não receberam uma forte base inicial de habilidades enfrentam um trade-off entre eficiência e equidade. Assim, investimentos na primeira infância possuem um importante argumento econômico que os justificam na hora da alocação de recursos para a política pública.

Mesmo quando as intervenções no início da infância não aumentam o QI, elas ampliam as habilidades não cognitivas, que também têm grandes efeitos na escolaridade, no mercado de trabalho e em outros aspectos comportamentais, como gravidez na adolescência. $E$ assim, por meio dos efeitos na escolaridade, essas intervenções podem elevar a pontuação em testes mesmo não aumentando o QI, o que demonstra a importância crucial das habilidades não cognitivas (CUNHA et al., 2006; GERTLER; FERNALD, 2004; GRACES et al., 2002). 


\section{A formação de habilidades no ciclo de vida e a escolha dos pais}

Cunha, Heckman e Schenach (2010) apresentam um modelo de formação de habilidades na primeira infância que incorpora os conceitos de autoprodutividade e complementaridade, contemplando, ainda, a importância de ambas habilidades cognitivas e não cognitivas na produção de resultados como escolaridade e salários, por exemplo. Nesta seção, o objetivo é modelar a escolha enfrentada pelos pais quando decidem colocar seus filhos no ensino infantil ou apenas no ensino fundamental (quando este é obrigatório) e os efeitos que essa escolha produzem sobre a formação das habilidades.

A partir da hipótese de que existe seleção por variáveis observáveis na escolha dos pais entre colocar ou não o filho no ensino infantil, este trabalho utiliza o método de pareamento propensity score matching, para criar grupos de comparação com respeito a essas características que parecem influenciar na escolha.

Considere-se um caso de uma família representativa em que a única forma de herança é a educação. Para simplificar o problema, suponha-se que existam apenas dois tipos de educação: a infantil e o ensino fundamental, $p_{1}$ e $p_{2}$, respectivamente. Os indivíduos altruístas acumulam capital humano com a educação recebida, de acordo com a seguinte função: $H_{i}\left(p_{1}, p_{2}\right)=a^{p_{1}} p_{2}$

O ensino fundamental é o único obrigatório, assim, $p_{2}$ é uma variável exógena, e os indivíduos escolhem se alocam seus recursos em $p_{1}$. A educação infantil é discreta e pode ser 2 ou 0 . Se os pais decidem alocar $p_{1}=2$, então aumentam o capital humano dos filhos em $a^{2}$. Se alocam $p_{1}=0$, então o capital humano dos filhos será determinado apenas por $p_{2}$, ou seja, somente o ensino fundamental. A acumulação do capital humano determina a renda dos indivíduos $Y\left(H_{i}\left(p_{1}, p_{2}\right)\right)$, que pode ser alocada entre consumo e o gasto no capital humano de seus filhos, conforme a equação (2):

$Y\left(H_{p}\left(p_{1}, p_{2}\right)\right)=u(c)+H_{f}\left(p_{1}, p_{2}\right)$

Onde $p$ e $f$ representam a função para os pais e a função para os filhos, respectivamente. Assim, a resolução do problema de maximização da utilidade do indivíduo fica:

$u(c)+H_{f}\left(p_{1}, p_{2}\right)-\lambda Y\left(H_{p}\left(p_{1}, p_{2}\right)\right)$

Resolvendo o lagrangiano:

$$
\begin{aligned}
& \frac{d L}{d c}=u^{\prime}(c) \\
& \frac{d L}{d p_{1}}=H_{f}^{\prime}\left(p_{1}, p_{2}\right)-\lambda Y^{\prime}\left(H_{p}\left(p_{1}, p_{2}\right)\right)=0 \\
& H_{f}^{\prime}\left(p_{1}, p_{2}\right)=\lambda Y^{\prime}\left(H_{p}\left(p_{1}, p_{2}\right)\right)
\end{aligned}
$$


A equação (6) mostra que a acumulação do capital humano herdado pelo filho depende do capital humano que recebeu do pai e da renda que esse capital humano the permite receber. Ou seja, a escolha entre colocar ou não o filho no ensino infantil depende de duas variáveis: o capital humano dos pais (medida aqui como escolaridade); e a renda dos pais (medida por variáveis de bens de consumo). Consideram-se agora o caso do pai que recebeu educação infantil (7) e o caso do pai que recebeu apenas o ensino fundamental (8):

$H_{f}^{\prime}\left(p_{1}, p_{2}\right)=\lambda a^{p_{1}-1} p_{1} p_{2}$

$H_{f}^{\prime}\left(p_{1}, p_{2}\right)=\lambda p_{2}$

A equação (8) é menor do que a (7); portanto, o pai que recebeu uma educação menor, neste caso não fez o ensino infantil, tende a ter uma renda menor, o que fará com que, no equilíbrio da maximização da sua utilidade, ele não invista na educação infantil dos seus filhos, gerando um círculo vicioso, em que famílias com baixa escolaridade tenderão a ter filhos com escolaridade e, por conseguinte, renda baixas. Menezes Filho (2007) e Soares e Collares (2006) apresentam evidências que sustentam a hipótese de que escolaridade importa nessa escolha.

Existem ainda algumas variáveis importantes na seleção, e que por simplificação não aparecerem no modelo, mas que não são diretamente observáveis, como a disponibilidade de escola nas proximidades onde o aluno mora, interesse ou valorização dos pais pela educação de seus filhos e a qualidade da educação, representando um dos limites deste trabalho. Em estudo com dados de 147 instituições de educação infantil em seis capitais brasileiras, Campos et al. (2011) mostraram que creches e pré-escolas apresentam, em média, níveis de qualidade insatisfatórios. 0 estudo ainda observa que algumas das variáveis associadas à maior qualidade referem-se a características socioeconômicas do bairro e da clientela das instituições. Ou seja, além do acesso ser mais difícil para alunos de baixo nível socioeconômico, a qualidade da educação oferecida tende a ser pior.

Outro aspecto importante que não aparece no modelo é a disponibilidade de alguém em casa para tomar conta do filho. Apesar de não ser diretamente observável, a variável possuir empregada foi utilizada como proxy e se ajustou bem ao modelo empírico.

\section{Metodologia utilizada no trabalho}

Esta seção apresenta brevemente as metodologias utilizadas no presente estudo, a saber, o método de pareamento propensity score matching (PSM) e o estimador linear duplamente robusto, mínimos quadrados ponderados pelo propensity score.

O estimador propensity score matching (PSM)

Considerando a hipótese de seleção por variáveis observáveis na escolha entre frequentar ou não a educação infantil, o método de propensity score matching tenta definir 
um grupo de controle para comparação, modelando a probabilidade de participar do tratamento conforme uma função de características observadas $X$ que contém toda a informação desse vetor, o qual é chamado de propensity scores ou escores de propensão. Assim, nesse método, o pareamento entre o grupo tratado e o de controle é baseado nessa probabilidade, que pode ser calculada por meio de um modelo probit (ROSENBAUM; RUBIN, 1983).

Dessa forma, o efeito médio do tratamento corresponde à diferença dos resultados médios entre os dois grupos - tratamento e controle. No entanto, a validade deste método depende das seguintes hipóteses:

- hipótese de seleção nos observáveis ou ignorabilidade: o vetor de variáveis observáveis $X$ contém todas as informações sobre o resultado potencial na ausência do tratamento $(Y(0))$ que o indivíduo possui ao tomar a decisão de participar ou não do tratamento. Isso significa que não há nada sistemático que faça com que um indivíduo seja ou não tratado, sendo que a variável $Y(0)$ torna-se independente de T. Formalmente define-se como:

$\left(Y_{i}(0) Y_{i}(1)\right) \perp T_{i} \mid p\left(X_{i}\right)$

- hipótese de sobreposição: esta condição garante que cada indivíduo no grupo tratamento tenha um par próximo de comparação no grupo de controle na distribuição do escore de propensão e no qual a variável resultado corresponderia à situação deste indivíduo na ausência do tratamento. Esta hipótese define-se como:

$0<\operatorname{Pr}[T=1 \mid X]=p(X)<1$

Uma das críticas aos métodos baseados nestas duas hipóteses principais é que, ao tomarem a decisão de participar ou não, os indivíduos também levam em consideração características não observáveis. Cumpridas as hipóteses de ignorabilidade e sobreposição, a média dos resultados dos não tratados pareados com os grupos identifica a média contrafactual resultante no grupo tratado na ausência de tratamento (BECKER; ICHINO, 2002).

O estimador de mínimos quadrados ponderados pelo propensity score

Segundo Imbens e Wooldridge (2008), estimativas de efeitos controlando para características observáveis com o estimador de mínimos quadrados ponderados pelos escores de propensão, ou como chamado aqui MQP, contornam o problema de má especificação, por ser uma combinação do propensity score com a regressão, que tem o benefício de ser duplamente robusto. Esse problema pode estar na equação da regressão ou do propensity score, mas nunca em ambos. Assim, estes métodos chamados como duplamente robustos removeriam o efeito direto das variáveis omitidas na regressão e reduziriam a correlação entre as variáveis omitidas e incluídas, reponderando-as.

Segundo Becker e Ichino (2002), para a realização do mínimos quadrados ponderados pelos escores de propensão, são necessários dois procedimentos: o primeiro é o cálculo dos escores de propensão, que pode ser feito por meio de um modelo probit ou logit. No segundo 
procedimento, estima-se o mínimos quadrados ponderados pelos escores de propensão propriamente dito. Essa combinação de métodos estima uma regressão ponderada com o peso igual a 1 para os indivíduos do grupo tratamento e igual a $\frac{\hat{P}\left(X_{i}\right)}{1-\hat{P}\left(X_{i}\right)}$ para os indivíduos do grupo de controle (PINTO, 2012). A regressão estimada e as funções de pesos são:

$Y i=\alpha+\beta^{\prime X i}+\tau . W i+\varepsilon_{i}$

$\lambda_{i}=\sqrt{\frac{W_{i}}{e\left(X_{i}\right)}+\frac{1-W_{i}}{1-e\left(X_{i}\right)}}$

Onde:

$Y i=$ resultado;

$W i=$ variável de participação no programa (0 ou 1);

$e\left(X_{i}\right)=$ propensity score estimado;

$X_{i}=$ características observadas que explicam a participação no programa;

$\lambda_{i}=$ peso.

0 coeficiente $\tau$ estimado é um estimador consistente para o efeito médio do tratamento (ATE). Essa regressão, além de diminuir o viés, aumenta a precisão da estimativa. Assim, com os pesos dados pelos escores de propensão, o estimador de mínimos quadrados se torna consistente. Sendo ê um estimador consistente do propensity score e garantindo que cada termo dentro da raiz quadrada some 1 , o estimador por mínimos quadrados ponderados é consistente para o ATE:

$\hat{\lambda}_{i}=\sqrt{\frac{W_{i} / \hat{e}\left(X_{i}\right)}{\sum_{i=1}^{N} W_{i} / \hat{e}\left(X_{i}\right)}+\frac{\left(1-W_{i}\right) /\left(1-\hat{e}\left(X_{i}\right)\right)}{\sum_{i=1}^{N}\left(1-W_{i}\right) / 1-e\left(X_{i}\right)}}$

Para se estimar o efeito tratamento sobre os tratados (ATT), o ponderador produz o estimador do coeficiente $\tau$ que é consistente para o ATT:

$\hat{\lambda}_{i}=\sqrt{\frac{W_{i} / \hat{e}\left(X_{i}\right)}{\sum_{i=1}^{N} W_{i}}+\frac{\left(1-W_{i}\right) \hat{e}\left(X_{i}\right) /\left(1-\hat{e}\left(X_{i}\right)\right)}{\sum_{i=1}^{N}\left(1-W_{i}\right) \cdot \hat{e}\left(X_{i}\right) / 1-e\left(X_{i}\right)}}$

Esta análise de ponderação garante que as covariâncias não sejam correlacionadas com o indicador de tratamento, gerando, assim, um estimador consistente. Dessa forma, a adição das covariâncias no modelo estimado aumenta a precisão das estimativas.

\section{Base de dados e estratégia empírica}

Esta seção apresenta uma breve explanação da base de dados utilizada e a estratégia empírica deste trabalho.

A base de dados: Prova Brasil 2011

Desenvolvida pelo Instituto Nacional de Estudos e Pesquisas Educacionais Anísio Teixeira (Inep/MEC), a Prova Brasil e o Sistema Nacional de Avaliação da Educação Básica (Saeb) têm o objetivo de avaliar a qualidade do ensino no Brasil, utilizando testes 
padronizados e questionários socioeconômicos. Esses testes são aplicados no $5^{\circ}$ e e $9^{\circ}$ ำ anos do ensino fundamental das escolas públicas que possuíam no mínimo 20 alunos matriculados em cada ano avaliado, nas redes estaduais, municipais e federal, de áreas rural e urbana. Os testes levam em consideração os conhecimentos dos alunos em matemática e língua portuguesa, e os questionários abrangem informações socioeconômicas, que ajudam no processo de relacioná-las com o desempenho dos estudantes. É importante salientar que os questionários são respondidos pelos próprios alunos, que podem desconhecer ou responder erroneamente alguma questão mais complexa, o que constitui uma das limitações deste tipo de banco de dados.

Mais do que dados oriundos dos alunos, a Prova Brasil coleta informações relacionadas aos professores e diretores, como condições de trabalho, dados demográficos e perfil profissional. Além de auxiliarem no aprimoramento da qualidade da educação e redução das desigualdades, as médias de desempenho subsidiam o cálculo do Índice de Desenvolvimento da Educação Básica (Ideb), e estão disponíveis para o público, o que pode ser muito útil, já que os dados são comparáveis ao longo do tempo e ampliados a cada dois anos.

O banco de dados apresenta informações de 1.792 .242 alunos, 5.296 municípios e 40.799 escolas, para o 5을 ano e 1.984.181 alunos, 5.458 municípios e 31.599 escolas, para o $9^{0}$ ano. As variáveis resultado utilizadas na estimação são as notas em português e matemática para o 5ํㅜ e 9ํaa anos, mensuradas na escala Saeb.

Para $05^{0}$ ano, a distribuição das notas de português apresenta uma média de 192 pontos na escala única do Saeb - a Prova Brasil utiliza a mesma metodologia do Saeb, baseada na teoria de resposta ao item (TRI), o que permite a comparação entre as notas -, máximo de 339 pontos e mínimo de 77 pontos. 0 desvio padrão para a nota de português é de 45 pontos. Já a distribuição de notas em matemática tem uma média de 211 pontos na escala única do Saeb, máximo de 338 pontos e mínimo de 90 pontos, com desvio padrão de 46 pontos.

Para o 9ํano, a nota em português possui média de 239 pontos na escala única do Saeb, com máximo de 380 pontos, mínimo de 103 pontos e desvio padrão de 46,7 pontos. Já a nota em matemática tem média de 245 pontos na escala única do Saeb, máximo de 398 pontos, mínimo de 105 pontos e desvio padrão de 47 pontos.

\section{Estratégia empírica}

Na estimação do pareamento por propensity score matching (PSM), o grupo de tratamento é formado pelos alunos que frequentaram o ensino infantil (um tratamento para os que iniciaram na creche e outro para os que começaram na pré-escola) e o grupo de controle por aqueles que iniciaram diretamente no ensino fundamental. No questionário da Prova Brasil 2011, a pergunta é quando o aluno iniciou os estudos, se creche, pré-escola ou diretamente no primário. Essa, portanto, foi a variável tratamento utilizada no modelo. ${ }^{9}$

\footnotetext{
$\overline{9}$ É razoável supor que os alunos que entraram na creche continuaram na pré-escola e depois no ensino fundamental.
} 
Segundo Crump, Hotz, Imbens e Mitinik (2009, 2008), o estimador de pareamento é muito sensível à escolha das variáveis $X$. Desse modo, um número muito grande de variáveis explicativas pode invalidar a hipótese de sobreposição, enquanto um número muito pequeno pode violar a hipótese de ignorabilidade. Para cumprir a hipótese de sobreposição, foram determinados grupos de variáveis como proxy para as variáveis de seleção apresentadas no modelo teórico de escolha, ${ }^{10}$ para que existisse alguma maleabilidade de escolha de variáveis que satisfizessem as suposições do modelo empírico.

O primeiro passo para o cálculo do PSM é a estimação de um modelo probit que busca identificar, por meio das variáveis observáveis citadas - renda, escolaridade dos pais e disponibilidade de alguém em casa para cuidar da criança -, a probabilidade de o aluno ter frequentado o ensino infantil.

Assim, foram duas estimativas e, portanto, duas variáveis de tratamento diferentes: ter iniciado os estudos na creche e ter iniciado os estudos na pré-escola, para cada ano. Para ambos os modelos, o grupo de controle foi ter iniciado diretamente no ensino fundamental.

Após os pareamentos, foram estimadas regressões medindo os efeitos médios do tratamento (ensino infantil) no desempenho dos alunos em português e matemática, para cada ano, a saber $5^{\circ}$ e $9^{0}$ anos do ensino fundamental. Essas regressões foram estimadas por meio do método dos mínimos quadrados ponderados pelo propensity score, levando em conta as características observáveis de seleção (contidas nos escores de propensão) e características com relação ao desempenho, como a raça do aluno (SOARES; ALVES, 2003), fatores culturais como frequentar a biblioteca, ler regularmente e fazer o dever de casa de português e de matemática, o fato de ter sido reprovado, ou ter sempre estudado em escola pública, de já ter abandonado a escola alguma vez e de trabalhar fora (SOARES; COLLARES, 2006; MENEZES FILHO, 2007).

\section{Análise dos resultados}

Como explanado na estratégia empírica, duas estimativas, para creche e pré-escola, foram feitas para cada questionário da Prova Brasil do $5^{\circ}$ e 9 ํㅡㅁos. Os resultados dos modelos probit mostraram que todas as variáveis incluídas no modelo são estatisticamente significantes a $1 \%$. Os sinais dos coeficientes indicam haver uma relação positiva entre a renda e a escolaridade dos pais na probabilidade de frequentar o ensino infantil, seja na creche ou na pré-escola. Isso evidencia que a seleção por observáveis como renda e escolaridade dos pais realmente se verifica empiricamente. A proxy para disponibilidade de alguém em casa para cuidar da criança, que é ter empregada, só foi significante para

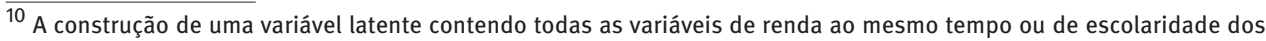
pais, por exemplo, algumas vezes impossibilitou a hipótese de sobreposição (balanceamento). Portanto, para a renda, por exemplo, foram utilizadas algumas variáveis binárias de bens de consumo e, para escolaridade, variáveis binárias como a mãe ter ensino superior completo e saber ler. Foi utilizado o máximo possível de variáveis que cumprissem as suposições do modelo. Assim, foi possível cumprir a condição de balanceamento para todas as estimações, tendo em todos os modelos pelo menos um componente de cada variável teórica apresentada no modelo da terceira seção.
} 
as estimativas da creche e, desse modo, ela não foi incluída nos modelos da pré-escola. A Tabela 1 apresenta os resultados das estimativas pelo método dos mínimos quadrados ordinários ponderados pelos escores de propensão, e seus respectivos testes de significância.

TABELA 1

Estimativa do efeito médio do tratamento nos tratados pelo método dos mínimos quadrados ponderados pelo propensity score

Brasil - 2011

\begin{tabular}{|c|c|c|c|c|}
\hline Estimativa & № de tratados & № controles & ATT - MQP & $\tau$ \\
\hline Efeito creche na nota de português - 5ํano & 349.081 & 573.450 & 5.063 & 34.04 \\
\hline Efeito creche na nota de matemática - 5ํano & 349.081 & 573.450 & 5.735 & 36.99 \\
\hline Efeito pré-escola na nota de português - $5^{\circ}$ ano & 373.786 & 588.624 & 11.213 & 83.33 \\
\hline Efeito pré-escola na nota de matemática - 5oano & 373.786 & 588.624 & 12.497 & 88.88 \\
\hline Efeito creche na nota de português - $9^{0}$ ano & 496.409 & 1.023 .064 & 4.094 & 35.84 \\
\hline Efeito creche na nota de matemática - 9ํano & 496.409 & 1.023 .064 & 3.521 & 30.60 \\
\hline Efeito pré-escola na nota de português - $9^{0}$ ano & 592.659 & 660.621 & 11.010 & 96.00 \\
\hline Efeito pré-escola na nota de matemática - 9oa ano & 592.659 & 660.621 & 10.261 & 88.36 \\
\hline
\end{tabular}

Fonte: Instituto Nacional de Estudos e Pesquisas Educacionais Anísio Teixeira - Inep. Prova Brasil 2011. Nota: Dados estimados por meio do software stata 11.

Para todas as estimativas, o efeito do ensino infantil se mostrou positivo e estatisticamente significante em comparação com os alunos que entraram diretamente no ensino fundamental. 0 efeito da pré-escola nas notas do 50 ano - os maiores impactos em magnitude estão no $5^{\circ}$ ano -,se mostrou positivo e altamente significante, aumentando em 11,2 e 12,5 pontos na escala Saeb em português e matemática, respectivamente. Isso significa que, para a nota de português, para um desvio padrão de 45 pontos, um aluno na mediana da distribuição passaria à frente de $10 \%$ dos alunos apenas por ter frequentado a pré-escola. Para a nota de matemática, isso se traduz para um aluno da mediana passar à frente de $11 \%$ da distribuição apenas por ter frequentado a pré-escola.

Um aluno do 5ำ ano teria, em média, 192 pontos na escala Saeb em português e estaria no nível 3, sendo capaz de competências como identificar os elementos da narrativa em letras de música e fábulas, reconhecer a finalidade de abaixo-assinado e verbetes e as relações entre pronomes e seus referentes e causa e consequência em fragmentos de romances, diários, crônicas, reportagens e máximas (provérbios), interpretar o sentido de conjunções, de advérbios, e as relações entre elementos verbais e não verbais em tirinhas, fragmentos de romances, reportagens e crônicas e comparar textos de gêneros diferentes que abordem o mesmo tema, dentre outras competências.

Esse mesmo aluno, apenas por ter frequentado a pré-escola, se deslocaria para o nível 4, em que, além dessas competências, seria capaz de identificar informação explícita em sinopses e receitas culinárias, assunto principal e personagem em contos e letras de música, formas de representação de medida de tempo em reportagens, efeito de humor em piadas, sentido de expressão, elementos da narrativa e opinião e relação de causa e consequência e entre pronomes e seus referentes em fábulas, poemas, contos e tirinhas. 
O efeito da creche no 5ㅇ ano é um pouco mais discreto, sendo de 5,06 e 5,73 em português e matemática, respectivamente.

A Tabela 1 apresenta também os efeitos para os alunos do 9 음 ano. Aqueles que frequentaram a pré-escola possuem um desempenho de 11,01 e 10,26 pontos a mais em português e matemática, respectivamente, em relação aos que entraram diretamente no ensino fundamental. Já para a creche, esse efeito é de 4,09 e 3,52, para português e matemática, respectivamente. 0 menor efeito da creche em matemática, para um desvio padrão de 47 pontos, significa que um aluno na mediana da distribuição ultrapassaria 3\% da distribuição apenas por ter frequentado a creche.

Além do retorno em termos de melhor desempenho dos alunos, os investimentos em educação, especialmente na primeira infância, apresentam altos retornos econômicos e sociais. Os primeiros podem ser mensurados, já os outros tipos de retornos e externalidades positivas que a educação pode proporcionar são difíceis de medir. No entanto, há diversas evidências de que esses retornos são muito altos (GROSSMAN, 2006 apud BARBOSA FILHO; PESSOA, 2009).

É possível argumentar também que esses resultados podem estar subestimando o verdadeiro impacto do ensino infantil, uma vez que, além de aumentos nas habilidades cognitivas, as evidências em estudos internacionais mostram que as habilidades não cognitivas, que também são responsáveis pelos altos resultados educacionais e no mercado de trabalho, são amplamente desenvolvidas no ensino infantil. Trabalhos futuros têm como importante tarefa estimar evidências da magnitude dos efeitos do ensino infantil sobre as habilidades não cognitivas, bem como viabilizar a medição dessas habilidades.

\section{Conclusão}

Este trabalho estimou o impacto da frequência no ensino infantil sobre o desempenho dos alunos no ensino fundamental de $5^{\circ}$ e 90 anos, em português e matemática na Prova Brasil 2011, considerando o problema da seleção por variáveis observáveis que parece ocorrer na escolha dos pais em colocarem seus filhos no ensino infantil ou diretamente no fundamental, e características que afetam o desempenho dos alunos.

Na primeira metodologia utilizada, que diz respeito ao problema de seleção, o método propensity score (MQP-PS), que cria grupos comparáveis entre si, foi estimado por meio da hipótese de que a escolha dos pais entre colocar ou não seu filho no ensino infantil parece ser majoritariamente explicada por três aspectos: capital humano dos pais; renda ou nível socioeconômico; e disponibilidade de alguém para cuidar da criança em casa. Foram utilizadas como proxies variáveis disponíveis na base de dados da Prova Brasil 2011.

Para controlar também as variáveis que influenciam no desempenho dos alunos, a segunda metodologia empregada foi a regressão linear por mínimos quadrados ponderados pelo propensity score (MQP-PS). Esse método estima uma regressão em que a variável dependente é o desempenho do aluno e as independentes são, além da variável de interesse 
(a frequência no ensino infantil), outras variáveis que podem influenciar no desempenho dos alunos, como a raça, fatores culturais como frequentar a biblioteca, ler regularmente e fazer os deveres de casa e informações como ter reprovado ou ter sempre estudado em escola pública (SOARES; ALVES, 2003; SOARES; COLLARES, 2006; MENEZES FILHO, 2007). Esse método é duplamente robusto, pois, além de controlar as variáveis de desempenho, pondera a regressão pelos escores de propensão, que contêm a probabilidade de o aluno ter iniciado seus estudos no ensino infantil, dadas as variáveis do modelo de escolha dos pais.

A principal contribuição deste trabalho foi utilizar um método quase experimental aliado a um método duplamente robusto, buscando-se controlar o viés de seleção e o viés pelo background do aluno, simultaneamente. Os resultados estimados para creche e pré-escola mostraram um impacto positivo e significante estatisticamente tanto para as notas de português quanto para as de matemática de ambas as séries. Estes resultados são condizentes e complementam aqueles encontrados por Curi e Menezes Filho (2006), que previram efeitos sobre a conclusão das etapas de ensino e sobre os salários futuros, complementando o impacto também sobre as habilidades cognitivas no período do ensino fundamental.

Os resultados são também condizentes com Felício e Vasconcellos (2007), que identificaram efeitos no Saeb 2003 nas notas de matemática do 5 ํano, evidenciando que os efeitos se mantêm para o 9 음 ano e em notas de testes padronizados de português, assim como encontrado para dados argentinos (BERLINKSI et al., 2006) e países desenvolvidos (FEINSTEIN et al., 1998).

\section{Referências}

BARBOSA FILHO, F. H.; PESSOA, S. Educação, crescimento e distribuição de renda: a experiência brasileira em perspectiva histórica. In: VELOSO, F.; PESSOA, S.; HENRIQUES, R.; GIAMBIAGI, F. (Org.). Educação básica no Brasil. São Paulo: Elsevier Editora Ltda., 2009.

BECKER S.O.; ICHINO, A. Estimation of average treatment effects based on propensity score. Stata Journal, v. 2, n. 4, p. 358-377, 2002.

BERLINSKI, S.; GALIANI, S.; GERTLER, P. The effect of pre-primary education on primary school performance. An Arbor: William Davidson Institute, 2006 (Working paper, n. 838).

CAMPBELL, F. A.; RAMEY, C. T.; PUNGELLO, E.; SPARLING, J.; MILLER-JOHNSON, S. Early childhood education: Young adult outcomes from the Abecedarian Project. Applied Developmental Science, v. 6, n. 1, p. 42-57, Jan. 2002.

CAMPOS, M. M. Educação infantil: o debate e a pesquisa. Cadernos de Pesquisa, São Paulo, n. 101, p. 113-127, jul. 1997.

CAMPOS, M. M.; ESPOSITO Y. L.; BHERING, E.; GIMENEZ, N.; ABUCHAIM, B. A qualidade da educação infantil: um estudo em seis capitais brasileiras. Cadernos de Pesquisa, São Paulo, v. 41, n. 142, p. 20-54, jan./abr. 2011.

CARNEIRO, P.; HECKMAN, J. J. The evidence on credit constraints in post-secondary schooling. Economic Journal, v. 112, n. 482, p. 705-734, 2002. 
Human capital policy. In: HECKMAN, J.J.; KRUEGER, A. B.; FRIEDMAN (Ed.). Inequality in America: what role for human capital policies? Cambridge, MA: MIT Press, 2003.

CUNHA, F.; HECKMAN J.; LOCHNER, L.; MASTEROV, D. Interpreting the evidence on life cycle skill formation. In: HANUSHEK, E.; WELCH, F. (Ed.). Handbook of the economics of education. Amesterdã: Elsevier, 2006. v. 1, p. 695-812.

CURI, A.; MENEZES FILHO, N. Os efeitos da pré-escola sobre os salários, a escolaridade e a proficiência escolar. In: ENCONTRO NACIONAL DE ECONOMIA, 34. Anais... Salvador: Anpec, 2006.

FEINSTEIN, L.; ROBERTSON, D.; SYMONS, J. Pre-school education and attainment in the NCDS and BCS. London, UK: Centre for Economic Performance, London School of Economics and Political Science, 1998 (CEP Discussion Paper, n. 382).

FELÍCIO, F.; VASCONCELLOS, L. O efeito da educação infantil sobre o desempenho escolar medido em exames padronizados. In: ENCONTRO NACIONAL DE ECONOMIA, 35. Anais... Recife: Anpec, 2007.

GERTLER, P. J.; FERNALD, L. C. The medium term impact of oportunidades on child development in rural areas. 2004. Unpublished manuscript.

GRACES, E.; THOMAS, D.; CURRIE, J. Longer-term effects of head start. The American Economic Review, v. 92, n. 4, p. 999-1012, 2002.

GUIMARÃES, J. L.; PINTO J. M. R. A demanda pela educação infantil e os recursos disponíveis para seu financiamento. Em Aberto, v. 18, n. 74, p. 12-105, jul. 2001.

HECKMAN, J. J. Policies to foster human capital. Research in Economics, v. 54, n. 1, p. 3-56, 2000.

Schools, skills, and synapses. Economic Inquiry, v. 46, n. 3, Jul. 2008.

HECKMAN, J.; MASTEROV, D. V. The productivity argument for investing in young children. National Bureau of Economic Research, 2007 (IZA Discussion Paper, n. 2725).

IMBENS, G. M.; WOOLDRIDGE, J. M. Recent development in the econometrics program evaluation. National Bureau of Economic Research, 2008 (Technical Working Paper, n. 14251).

KNUDSEN, E. I. Sensitive periods in the development of the brain and behavior. Journal of Cognitive Neuroscience, v. 16, n. 1, p. 1412-1425, 2004.

REYNOLDS A. J.; TEMPLE, J. A.; ROBERTSON, D. L.; MANN, E. A. Long-term effects of an early childhood intervention on educational achievement and juvenile arrest: a 15-year follow-up of low-income children in public schools. JAMA - Journal of the American Medical Association, 2001.

ROSENBAUM, P.; RUBIN. D. The central role of the propensity score in observational studies for causal effects. Biometrika, v. 70, n. 1, p. 41-44, Apr. 1983.

SCHWEINHART, L. J.; MONTIE, J.; XIANG, Z.; BARNETT, W. S.; BELFIELD, C. R.; NORES, M. Lifetime effects: the high/scope perry preschool study through age 40. Ypsilanti, Mich: High/Scope Press, 2005.

\section{Sobre os autores}

Walcir Soares da Silva Junior é doutorando do Programa de Pós-Graduação em Desenvolvimento Econômico da Universidade Federal do Paraná (UFPR). Realizou estágio no Institute of Education (University College London, Inglaterra), com bolsa Capes - PDSE. É mestre pelo Programa de Pós-Graduação em Desenvolvimento Econômico da UFPR e pesquisador do Núcleo de Avaliação de Políticas Públicas Educacionais (Nappe), da UFPR. 
Flávio de Oliveira Gonçalves é doutor em Economia pela Universidade de Brasília, visitou a Universita Cattolica Del Sacro Cuore di Milano (Doutorado sanduíche), Scuola Superiore Sant`Anna (Pós-Doutorado) e Universitá Bocconi. É professor da Universidade Federal do Paraná, com atuação no Programa de Pós-Graduação em Desenvolvimento Econômico.

\section{Endereço para correspondência}

Walcir Soares da Silva Junior

Rua Gov. Jorge Lacerda, 25, apto. 3, Guabirotuba

81510-040 - Curitiba-PR, Brasil

Flávio de Oliveira Gonçalves

Universidade Federal do Paraná, Setor de Ciências Sociais Aplicadas, Departamento de

Economia

Rua Prefeito Lothário Meissner, 632, Jardim Botânico

80210-170 - Curitiba-PR, Brasil

\section{Abstract}

Evidence of the relationship between the frequency in children's education and the performance of public elementary school students in Brazil

This paper aims to estimate the impact of the frequency of child education in the proficiency of public elementary school students in Brazil, using as proxy the scores of students in Portuguese and Math tests available in Prova Brasil 2011. The Propensity Score Matching methodology was used under the selection hypothesis by observable variables, which aims to find a counterfactual. In order to control characteristics related to the students' performance, linear regressions were estimated by the robust method of least squares weighted by propensity scores. The results point to positive and statistically significant effects on the grades of Portuguese and Math in the 5th and 9th grades for students who began in pre-school and for students who started in kindergarten compared with those who started directly in the primary school.

Keywords: Early childhood education. Propensity score matching. Least squares weighted by Propensity Score.

\section{Resumen}

La evidencia de la relación entre la frecuencia en la educación de los niños y el rendimiento de los estudiantes de la escuela primaria pública en Brasil

Este artículo tiene como objetivo estimar el impacto de la frecuencia de la educación infantil en la competencia de los estudiantes de la escuela primaria pública en Brasil, utilizando como proxy los puntajes de los estudiantes en portugués y matemáticas disponibles en la Prova Brasil 2011. Se utilizó la metodología de emparejamiento en las puntuaciones de propensión bajo el supuesto de selección de variables observables, cuyo objetivo es encontrar el contrafactual. Para controlar las funciones relacionadas con el rendimiento escolar, las regresiones lineales fueron estimadas por el método de mínimos cuadrados ponderados por las puntuaciones de propensión. Fueron encontrados efectos positivos y significativos estadísticamente en las notas 
de portugués y matemáticas del quinto y noveno grados para los alumnos que empezaron en la enseñanza preescolary para los estudiantes que comenzaron en el jardín infantil, en comparación con aquellos que comenzaron directamente en la primaria.

Palabras clave: Educación en primera infancia. Propensión score matching. Mínimos cuadrados ponderados por puntaje de propensión.

Recebido para publicação em 24/11/2015 Recomendado para publicação em 02/08/2016 Aceito para publicação em 09/09/2016 